\title{
AFM imaging of 3D conformations and surface energetics of reconstituted ion channels: mimicking lipid bilayer cell membrane
}

\author{
A.P. Quist, H. Lin, J. Thimm, A. Mechler, I. Doudevski, R. Lal \\ Neuroscience Research Institute, University of California, Santa Barbara, CA 93106, US.
}

Biomembranes are important interfaces in biological systems and determine formation, sustenance and death of virtually all multicellular living organisms. These biomembranes are dynamic nanostructures with complex multiple compartments of protein macromolecules embedded in lipidic bilayers. Interactions of these protein macromolecules with lipidic counterparts trigger an array of signal transduction pathways that underlie all functions of cells and tissue. In order to mimic and design complex biomaterials such as a cell membrane, we need to understand their molecular conformations and various physicochemical properties. We have used an array of techniques, including atomic force microscopy, electrophysiological recordings, biochemical assays to examine the structure of lipidic bilayer and vesicles. We then reconstituted an array of peptides, in monomeric as well as oligomeric forms in these vesicles and bilayers. Using the aforementioned techniques, we then examined the 3D molecular structure of the protein macromolecular complexes and examined the role of various lipids in their stability and conformations. We also examined the role of various forces and physical forces, including ionic charge, electrostatic field, van der Waals forces and hydration forces on the conformations of these macromolecules.

As examples of a range of protein macromolecules and their interaction with lipidic milieu, we present our results from two peptides, amyloid beta and connexins that differ in their size $(\sim 4 \mathrm{kD}$ vs. $\sim 40 \mathrm{kD}$ ), their primary and secondary structures, and in their physiological roles (protein misfolding diseases vs. cell-cell and cell-surround communication associated metabolic/ionic homeostasis).

We show, for the first time that amyloid peptides when reconstituted in appropriate lipid vesicles make calcium permeable channels. These channels allow uptake of ionic calcium and increase the stiffness of the vesicle by the energy generated by charge-charge interactions in a confined space (Figure 1) [1]. When reconstituted in lipid bilayers, these amyloids form ion-channels that induce ionic currents that are selective to cations (e.g., Ca) (Figure 2) [2]. Both vesicular calcium-uptake and channel ionic current are regulated by appropriate antagonists and blockers. The incorporation of amyloid in lipid membrane is modulated by the nature (and charge) of the lipids and cholesterol.

We show, for the first time that complex gap junctional hemichannels made of connexin 43 (cx43) form ion channels in appropriate lipid bilayer membrane (Figure 3) [3]. These ion channels undergo open and closed conformational changes in response to calcium, an important regulator of cell behavior. We also show that such conformational changes are effected by a change in the surface energetics that results from the rearrangement of hydrophobic peptides lining the mouth of the ion channels and that the conformational changes are explained by a complex model, other than the classic Poisson-Nernst-Plank model of electrical field across the lipid bilayer membrane.

Results for our work as well as our understanding of the model bilayers with well defined protein macromolecules are being used to design membranes with channels for therapeutic purposes. 


\section{References}

[1] S. Rhee et al., J. Biol. Chem. 273 (1998) 13379.

[2] H. Lin et al., FASEB J. 15 (2001) 2433.

[3] J. Thimm et al., J. Biol. Chem. (2005) in press.

[4] H. Lin et al., Biochem 38 (1999) 11189.

[5] A.P. Quist et al., J Cell Biol 148 (2000) 1063.

Acknowledgement: Supported by grants from NIH, NSF-NATO postdoctoral fellowship, Philip Morris external Grant Program,

Alzheimer's Association, and Dept of Heath California.
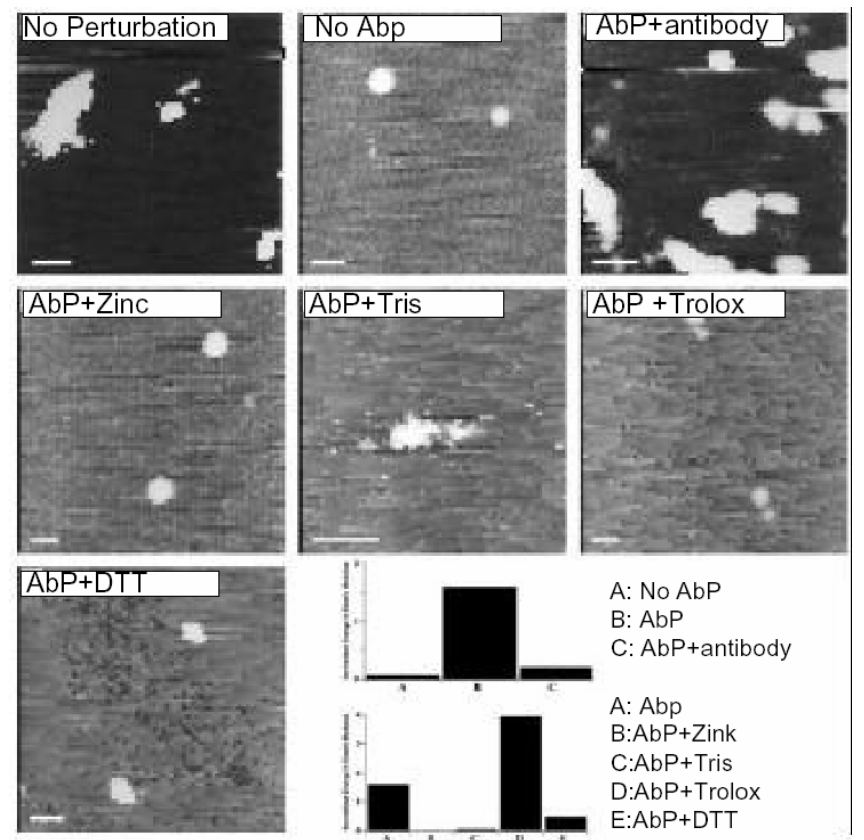

A: No AbP

B: AbP

C: AbP+antibody

\section{A: Abp}

$\mathrm{B}: \mathrm{AbP}+\mathrm{Zink}$

$\mathrm{C}: \mathrm{AbP}+$ Tris

$\mathrm{D}: \mathrm{AbP}+\mathrm{Trolox}$

Fig. 1. Height images of liposomes obtained during force mapping in non-calcium medium before adding any perturbation. Height images after adding perturbations and calcium have not changed from these pre-perturbation images. Histograms show normalized change in elastic modulus of the vesicles after adding perturbation and calcium [Ref 1].

\section{Amyloid beta protein forms ion channel}
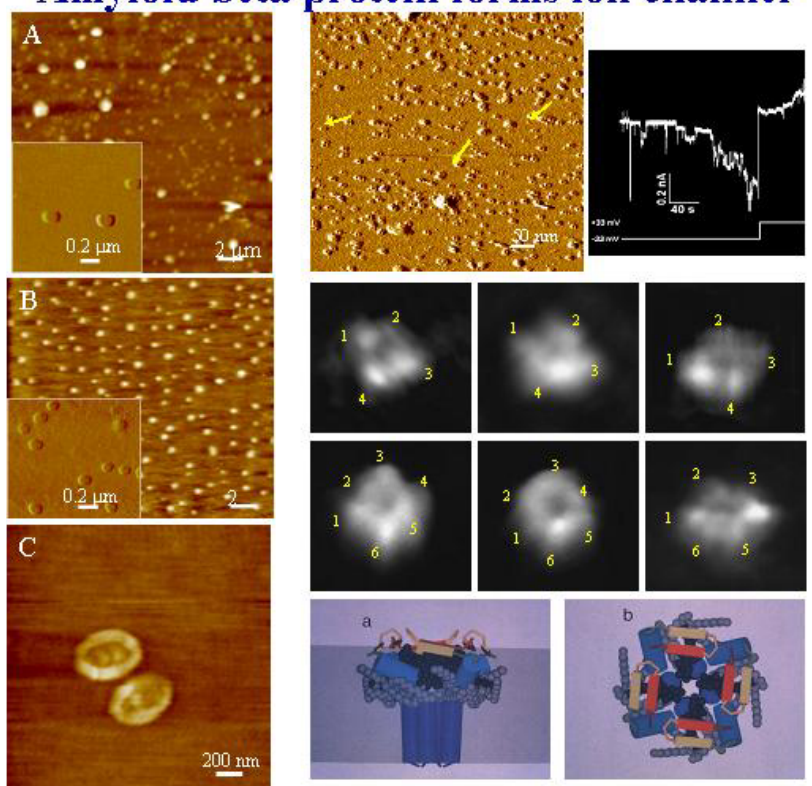

Fig. 2. Left: Liposomes reconstituted with (A) and without (B) $\mathrm{A} \beta \mathrm{P}(1-40)$, and dissected liposome reconstituted with $\mathrm{A} \beta \mathrm{P}(1-$ 40) (C).[Ref 4] Right: AFM image and electrical current record of $A \beta P(1-42)$ reconstituted in lipid bilayer (top). Individual $\mathrm{A} \beta \mathrm{P}(1-42)$ ion channels showing 4 and 6 subunits (middle). [Ref 2]. Molecular model of A $\beta$ P(1-42) ion channel (bottom) (From Durell et al, 1994).

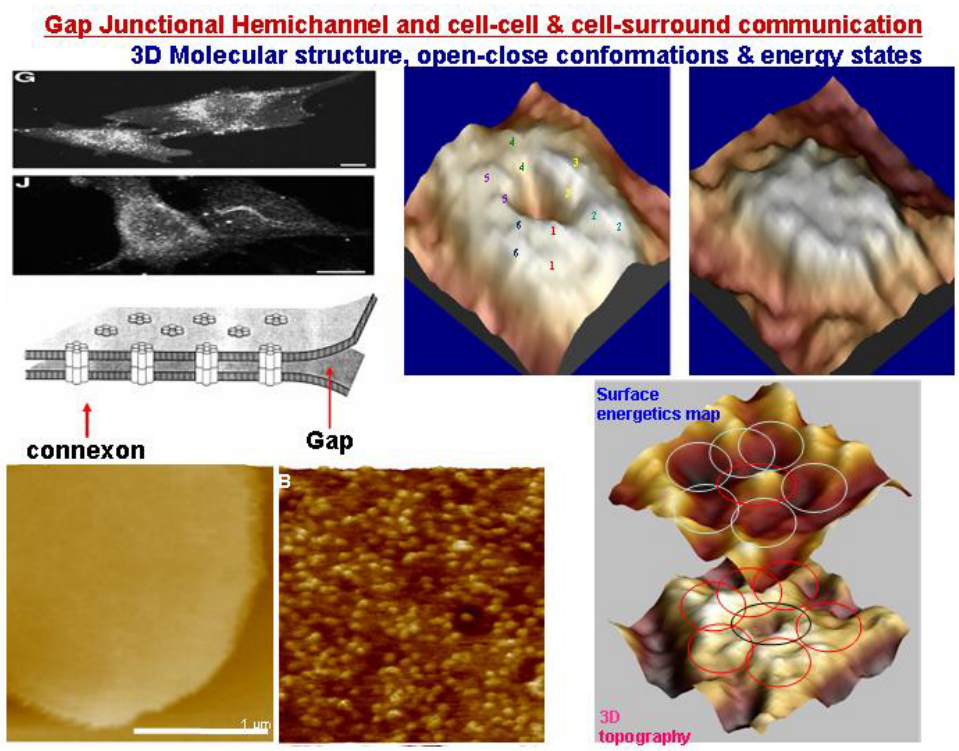

Fig. 3. Top Left: Immunofluorescence localization of Cx43 in fibroblast and N2A cell.[Ref 5]. Middle Left: schematic model of gapjuntions. Bottom Left: a large lipid bilayer membrane patch (bilayer height is $5 \mathrm{~nm}$ as determined by crosssection analysis, and a representative image of Cx43 hemichannels incorporated in the planar lipid bilayer reconstituted in calcium free buffer (scan size $400 \mathrm{~nm}$ ), no apparent crystalline cluster formation is observed.

Top Right: High resolution topography maps of connexin subunits of a single connexon. Left image shows a 3D height mode image of an open extracellular face of a hemichannel, imaged in nominally Ca-free buffer; right image shows a 3D height mode image of a closed hemichannel imaged in the presence of $1.8 \mathrm{mM} \mathrm{Ca} 2+$. Bottom Right: Surface energy map and 3D topography image of the same Cx43 hemichannel. [Ref 3] 